\title{
PRESENCIA DE LAS MUJERES EN LOS ÓRGANOS DE DIRECCIÓN DE LOS COLEGIOS PROFESIONALES DEL ÁMBITO DE LA SALUD EN 2015
}

\author{
Alicia Botello-Hermosa (1), Rosa Casado-Mejía (1) y Concepción Germán-Bes (2). \\ (1) Departamento de Enfermería. Facultad de Enfermería, Fisioterapia y Podología. Universidad de Sevilla. \\ (2) Departamento de Fisiatría y Enfermería. Facultad de Ciencias de la Salud. Universidad de Zaragoza.
}

\section{RESUMEN}

Fundamentos: La progresiva incorporación de las mujeres en las profesiones sanitarias no se corresponde con su presencia en puestos de responsabilidad. Dado que los colegios profesionales tienen un papel esencial en la representación y regulación profesional, se plantea como objetivo describir la frecuencia de la presencia de las mujeres en las estructuras directivas de los colegios profesionales del ámbito de la salud en España y comprobar el grado de cumplimiento de los criterios de paridad.

Métodos: Se identificó el género de la persona que ocupaba la presidencia, los cargos ejecutivos y la junta directiva visitando las webs del Consejo General de los colegios profesionales de Psicología, Consejo General de Colegios Oficiales de Médicos, Consejo General de Colegios Oficiales de Enfermería de España, Consejo General de Colegios de Fisioterapeutas, Consejo General de Dentistas, Organización Farmacéutica colegial y Consejo General de Colegios oficiales de Podólogos. Se describió el porcentaje de mujeres de forma global y según profesión y se comparó con las cifras de colegiados y colegiadas según el INE para 2014.

Resultados: De 251 colegios profesionales en julio de 2015, 41 $(21,91 \%)$ la presidencia estaba ocupada por mujeres. También ocupaban el $34,69 \%$ de los puestos ejecutivos y el $42,80 \%$ del total de las juntas directivas. Los colegios médicos y de enfermería tenían una mujer en la presidencia en el $11,32 \%$ y $43,48 \%$ respectivamente. Los de psicología fueron los que presentaron mayor presencia femenina en la presidencia, el 45,83\%.

Conclusión: No existe paridad entre hombres y mujeresen el conjunto de los colegios estudiados. La presencia femenina es mayor en colegios de psicología y enfermería y mucho menor en los de odontología, fisioterapia, podología y medicina con el nivel de responsabilidad disminuye la presencia de las mujeres.

Palabras clave: Sexismo. Personal sanitario. Mujeres médicas. Dentistas, mujeres. Enfermeras. Mujeres trabajadoras. Derechos de la mujer.

\section{Correspondencia}

Alicia Botello Hermosa

Departamento de Enfermería

Facultad de Enfermería, Fisioterapia y Podología

Universidad de Sevilla.

Avenzoar, 6

41009 Sevilla

abotello@us.es

DOI:

\section{ABSTRACT \\ Presence of Women in Management Bodies of the Professional Associations of the Field of Health in 2015}

Background: The gradual increase of women in the health professions does not correspond with her presence in positions of power. Given that professional colleges have an essential role in the representation and professional regulation, arises as an aim to describe the presence of women in the managerial structures of the professional colleges of health in Spain now to verify the degree of compliance with the criteria of parity.

Methods: The Spanish official professionals' colleges were compiled by visiting the websites of the General Council of the Psychology of Spain, General Council of Medical Associations of Spain, General Council of Colleges of Nursing of Spain, General Council of Physiotherapists Schools of Spain, General Dental Council, Organization collegiate Pharmaceutical General and Council of Associations of Podiatrists. All their webs were visited. The sex of the presidency, the executive and the entire board was identified. Data were analyzed according to the overall percentage of women and profession. We compared this to the INE-2014 collegiate professionals.

Results: Out of 251 professionals' colleges in July-2015, 21, 91\% had a female president. Women hold $34,69 \%$ of the executive positions and $42,80 \%$ of total boards. $11,32 \%$ of Medical colleges had a female president and $43,48 \%$ of Nursing ones. The Psychology are those with more women in the presidency, $45,83 \%$.

Conclusion: There is no parity, being higher in Psychology and Nursing and, much lower in Dentistry, Physiotherapy, Podiatry and Medicine. It decreases with the responsibility level. Health Inequality

Key words: Sexismo. Health personnel. Physicians women. Dentists, women. Nurses. Women, working. Women's rights. 


\section{INTRODUCCIÓN}

Los denominados "techos de cristal" impiden una igualdad real en la promoción de las mujeres a puestos de responsabilidad en nuestra sociedad ${ }^{1,2}$, aunque exista igualdad formal en el acceso a los mismos. Estas limitaciones invisibles han sido bien estudiadas en distintos ámbitos laborales ${ }^{1-3}$, también en el ámbito de la salud ${ }^{4,5}$.

Desde el siglo XX hay un progresivo aumento de las mujeres en medicina ${ }^{6}$ en particular y en las profesiones sanitarias en general que no se ha correspondido con la consiguiente representación femenina en los espacios de poder $^{7-10}$.

En este sentido, el informe de la Unidad de Mujeres y Ciencia, Científicas en Cifras $2013^{11}$, incide en la segregación horizontal de género en la profesión científica y en la formación así como en la segregación vertical en todos los sectores (universidades, organismos públicos de investigación, etcétera). De acuerdo con el estudio Las mujeres en los premios científicos en España 2009-2014², esto significa que "hay un estancamiento en la presencia de mujeres en los órganos de gobierno de las entidades científicas".

Los colegios profesionales tienen un papel esencial en la representación y en la regulación profesional en su ámbito de actividad ${ }^{13}$. Por tanto son de gran interés para estudiar las desigualdades de género en el contexto sanitario.

Teresa Ortiz et $\mathrm{al}^{6}$ en 2004 y Joana Morrison et al en $2010^{14}$ analizaron la representación de las mujeres en las sociedades científicas y revistas profesionales de Salud Pública, encontrando la existencia de procesos de segregación vertical que dificultaban la participación femenina en puestos de responsabilidad con un ligero aumento de participación durante el periodo 2000-2009. Consuelo Miqueo et a ${ }^{15}$ estudiaron la presencia de las mujeres en los comités de las revistas biomédicas encontrando un porcentaje bajo de las mismas y una opacidad de la mitad al no constar el nombre propio de los miembros de los comités directivos y consejo editoriales.

La Ley Orgánica 3/2007 para la igualdad efectiva de mujeres y hombres ${ }^{16}$, anima a adoptar medidas específicas en favor de las mujeres para corregir situaciones de desigualdad, como ocupar el $40 \%$ de los cargos de responsabilidad. En la Recomendación CM/ Rec (2007) ${ }^{17}$ del Comité de Ministros a los Estados miembros sobre las normas y los mecanismos de igualdad entre mujeres y hombres, se recuerda que todavía existen diferencias entre la igualdad de jure y la igualdad de facto. Así mismo, promover la igualdad real, incluyendo el empoderamiento de las mujeres es uno de los objetivos del Convenio de Estambul, ratificado por España y publicado en el BOE el 6 de junio de $2014^{17}$.

Todo ello hace necesario estudiar la relación entre la feminización de las profesiones sanitarias y los techos de cristal en el ámbito de las profesiones sanitarias, concretamente es importante realizar estudios en los que se describa la presencia de las mujeres en las estructuras directivas de los colegios profesionales de salud en España, poniendo de manifiesto la relación entre la feminización de las profesiones sanitarias y los techos de cristal en el ámbito de la salud, concretamente en los colegios profesionales, todo ello, con el fin de dar a conocer las necesidades e introducir las mejoras que eviten desigualdades e inequidades.

El objetivo de este trabajo fue describir la presencia de las mujeres en las estructuras directivas de los colegios profesionales del ámbito de la salud en España.

\section{MATERIAL Y MÉTODOS}

Se realizó un estudio descriptivo transversal. Se recopiló la información de todos los colegios profesionales del ámbito sanitario con representación provincial, autonómica o estatal a partir de 7 fuentes: Consejo General de la Psicología de España (http://www.cop. 
es/), Consejo General de Colegios Oficiales de Médicos de España (http://www.cgcom. es/), Consejo General de Colegios Oficiales de Enfermería de España (http://www.consejogeneralenfermeria.org/), Consejo General de Colegios de Fisioterapeutas de España (http://www.consejo-fisioterapia.org/), Consejo General de Dentistas (http://www. consejodentistas.es/), Organización Farmacéutica colegial (http://www.portalfarma. com/Paginas/default.aspx), Consejo General de Colegios oficiales de Podólogos (http:// www.cgcop.es/inicio/index.php) a fecha $1 /$ Junio/2015.

Se visitó la web de cada colegio profesional, descartando las no localizables ni por Internet ni telefónicamente. Se identificaron los siguientes cargos de juntas directivas: presidencia, vicepresidencia, secretaría, tesorería (considerados "ejecutivos") y vocales. Otros cargos que pudieran aparecer se asimilaban a los previamente definidos tras identificar el contenido de las funciones asignadas.

Según el nombre de quien ocupaba el cargo, se identificó su sexo. Si era equívoco, se buscó en Internet o se llamó telefónicamente al colegio profesional para aclararlo.

Se clasificaron por profesiones: enfermería, farmacia, fisioterapia, medicina, odontología, podología, y psicología.
La información sobre número de personas colegiadas se obtuvo de los datos del INE sobre de $2014^{18}$.

Se realizó una descripción global y de cada subgrupo del porcentaje de mujeres presentes en el conjunto de las juntas directivas así como en los puestos considerados "ejecutivos" y vocales.

\section{RESULTADOS}

Se identificaron 261 colegios profesionales de los que se descartaron 10 por no encontrarse información disponible sobre ellos. Se consideraron para la investigación la información relativa a 46 colegios profesioanles de enfermería, 49 de farmacia, 18 de fisioterapia, 53 de médicos, 45 de odontología, 24 de psicología y 16 de podología.

El total de mujeres en las juntas directivas de los 261 colegios profesionales del ámbito de la salud en España a 1 de junio de 2015 fue de $1.233(42,80 \%)$, pero al analizar por cargos se apreció que en cargos ejecutivos solo $400(34,69 \%)$ estaban ocupados por mujeres. El cargo con más mujeres eran las vocalías $833(48,07 \%)$, seguido por las secretarías $101(42,26 \%)$. El cargo con menos frecuencia ocupado por mujeres fue la presidencia 55 $(21,91 \%)$. En la tabla 1 se muestra la representación de mujeres estudiada por profesión.

\begin{tabular}{|l|r|r|r|r|r|r|r|r|r|}
\hline \multicolumn{7}{|c|}{ Tabla 1 } \\
\multicolumn{7}{|c|}{ Representación de las mujeres en las juntas directivas } \\
de los colegios profesionales españoles \\
\hline
\end{tabular}


Había una mujer ocupando la presidencia en en $1(2,22 \%)$ de los colegios de odontología, $1(6,25 \%)$ de podología, $2(11,11)$ de fisioterapeutas y $6(11,32 \%)$ de los colegios médicos, contrastando con $11(45,83 \%)$ de psicología o $20(43,48 \%)$ de enfermería. Esta diferencia disminuyó cuando se consideró el total de cargos ejecutivos, pasando a $41(43,84 \%)$ en odontología y $162(64,54 \%)$ en enfermería, por poner los casos más extremos (tabla 1). La recomendación legal de representación femenina del 40\% mínima se cumplió en el 51,73\% de los colegios profesionales estudiados.

\section{DISCUSIÓN}

La presencia de mujeres en cargos directivos de los colegios profesionales españoles no alcanza los niveles deseados. No se corresponde con el índice de paridad recomendado legalmente ni con la creciente feminización de estas profesiones, sin embargo sí presenta bastante similitud con la representación de las mujeres en las sociedades científicas de profesionales de la salud ${ }^{19}$.

La proporción de mujeres médicas en España va aumentando como lo indican los últimos datos del INE (48,4\% en 2014) ${ }^{18}$ o de las mujeres que se incorporan a una plaza MIR (un $65,9 \%$ en 2015) ${ }^{20}$, lo que contrasta con la baja representación de las mujeres en los colegios médicos. Se ha intentado explicar la escasa presencia de médicas en puestos de liderazgo bien a causa de los prejuicios hacia las aptitudes de las mujeres que desempeñan cargos de poder y liderazgo o por la cultura organizacional de las instituciones ${ }^{21}$. En esta tendencia de profesionales de la medicina se sitúan también profesiones de la salud más jóvenes, como la odontología, la fisioterapia o la podología que, estando feminizadas sus bases, mantienen la masculinización de los órganos de poder, además del sexismo en el lenguaje al nombrarlos (Consejo General de Colegios Oficiales de Médicos, Consejo General de Colegios oficiales de Podólogos). En los colegios de psicología, enfermería ${ }^{10} \mathrm{o}$ farmacia la representación femenina es ma- yor, pero nunca en el mismo porcentaje que sus bases. En la enfermería, históricamente femenina, cuando se produjo la unificación de colegios en 1978, los enfermeros han venido ocupando gran parte de los cargos directivos ${ }^{10}$.

Es de destacar la diferencia de representación femenina entre la presidencia y otros cargos como vocalías o secretarías, parece que se prefieren mujeres para cargos funcionales y de trabajo pero no para los que realmente significan representación.

Que la representación femenina en los colegios profesionales disminuya a mayor responsabilidad (menos presidentas que cargos "ejecutivos", que cargos en la Junta Directiva) y que ocurra igual que en las sociedades científicas de profesionales de la salud confirma la hipótesis que ya defendían Rosa Casado y Alicia Botello ${ }^{19}$ de que podría estar relacionado con el hecho de que en entornos más jerárquicos el poder masculino está más presente. Se puede afirmar apoyándose en la tesis de Ryene Eisler que jerarquía y patriarcado van de la mano ${ }^{22}$.

En casi la mitad $(48,27 \%)$ de colegios profesionales no se llega al $40 \%$ de representatividad recomendado, lo que implicaría, según la Ley, que deben contar con planes de equidad para reducir la discriminación directa o indirecta.

Se presenta la primera aproximación global de un análisis de género de los colegios profesionales en el ámbito de la salud en España. Se puede concluir que no existe paridad en el conjunto, la presencia femenina es mayor en los colegios de psicología, enfermería y farmacia y disminuye con el nivel de responsabilidad.

Aunque existen estudios que analizan y apuntan las causas de la escasa presencia femenina en espacios de poder en medicina o enfermería, no se han encontrado en relación con otras profesiones de la salud, lo que constituye una de sus principales limitaciones. 
Sería necesario repetir la descripción periódicamente para monitorizar la representación femenina en los colegios profesionales, analizar si tienen planes de equidad y seguirlos. También sería conveniente ampliarlo a otros puestos de responsabilidad tales como dirección de revistas científicas y equipos de redacción, presidencia de grupos de trabajos, comités de congresos, etcétera así como extenderlo a otros colectivos (sindicatos por ejemplo).

Sería interesante realizar un estudio cualitativo sobre los techos de cristal donde colegiados y colegiadas y cargos directivos de los colegios profesionales aporten sus explicaciones, sentimientos y percepciones sobre el tema.

Como conclusión se puede afirmar que, a la vista de los resultados, se evidencia que siete años después de aprobarse la Ley para la Igualdad Efectiva entre Mujeres y Hombres 3/2007 no se han conseguido cambios significativos en la presencia equilibrada de mujeres y hombres en los cargos de responsabilidad de los colegios profesionales sanitarios, a pesar de tratarse de colectivos feminizados.

Esto reafirma la necesidad de implementar acciones positivas que favorezcan la paridad en puestos de poder, ya que la propia dinámica social de una mayor incorporación de las mujeres en todos los sectores de la actividad no es suficiente para lograr dicho objetivo.

\section{BIBLIOGRAFÍA}

1. Tesch BJ, Wood HM, Helwing AL, Nattinger AB. Promotion of women physicians in academic medicine. Glass ceiling or stycky floor? JAMA.1995;273:1022-5

2. Guil A. Docentes e investigadoras en las universidades españolas: visibilizando techos de cristal. RIE. 2007;25:111-31

3. Alcalá P, Pérez Sedeño E, Santesmases MJ (coordinadoras). Mujer y Ciencia. La situación de las mujeres investigadoras en el sistema español de ciencia y tecnología. Madrid: Ministerio de Educación y Ciencia. Fundación Española para la Ciencia y Tecnología; 2005. Disponible en: www.fecyt.es/documentos/MUJERyCIENCIA_web. pdf
4. Colomer C, Peiró R. ¿Techos de cristal o escaleras resbaladizas? Desigualdades de género y estrategias de cambio en SESPAS. Gac Sanit. 2002;16:358-60

5. Carnes M, Morrissey C, Seller ES. Women's health and women's leadership in academic medicine; hitting the same glass ceiling? J Women's Health. 2008;17:1453-62

6. Ortiz-Gómez T, Birriel-Salcedo J, Ortega-del-Olmo R. Género, profesiones sanitarias y salud pública. Gac Sanit. 2004;18 Sup11:189-94

7. Arrizabalga P, Valls-Llobet C. Mujeres médicas: de la incorporación a la discriminación. Med Clin (Barc). 2005; $125: 103-7$

8. Heath I. Women in medicine: continuing unequal status of women may reduce the influence of the profession. BMJ. 2004;329:412-3

9. Wynn R. Saints and sinners: women and the practice of medicine throughout the ages. JAMA. 2000;283:668-9.

10. Celma M, Acuña A. Influencia de la feminización de la Enfermería en su desarrollo profesional. Rev Antropol Exp. 2009;9:119-36

11. Unidad de Mujeres y Ciencia. Secretaría de Estado de Investigación, Desarrollo e Innovación. Ministerio de Economía y Competitividad. Científicas en cifras 2013. Estadísticas e indicadores de la (des)igualdad de género en la formación y profesión científica, 2014. Madrid: Disponible online: http:/www.inmujer.gob.es/estadisticas/consulta.do?metodo=buscar

12. González Orta L. Las mujeres en los premios científicos en España 2009-2014. Madrid: Unidad de Mujeres y Ciencia de la Secretaría de Estado de Investigación, Desarrollo e Innovación del Ministerio de Economía y Competitividad; 2015.

13. Brennan MD, Monzon V. Professionalism: good for patients and healthcare organizations. Mayo Clin Proc. 2014;89:644-52.

14. Morrison J, Borrell C, Marí-Dell'Olmo M, Ruiz Cantero MT, Benach J, Fernández E et al. Desigualdades de género en la Sociedad Española de Salud Pública y Administración Sanitaria (2000-2009). Gac Sanit. 2010;24:334-8

15. Miqueo C, Germán-Bes C, Fernández-Turrado T, Barral MJ. Ellas también cuentan. Científicas en los comités de revistas biomédicas. Presencias de las mujeres en los comités de las revistas biomédicas. Zaragoza, Prensas Universitarias de Zaragoza PUZ, PUZ. 2011, 354 págs.

16. Boletín Oficial del Estado. Ley Orgánica 3/2007, de 22 de marzo, para la igualdad efectiva de mujeres y hombres. BOE núm. 71 de 23 de Marzo de 2007. 
17. Boletín Oficial del Estado. Instrumento de ratificación del Convenio del Consejo de Europa sobre prevención y lucha contra la violencia contra la mujer y la violencia doméstica, hecho en Estambul el 11 de mayo de 2011. BOE núm 137 de 6 de junio de 2014.

18. INE. Profesionales sanitarios colegiados 2014. Madrid: INE; 2015

19. Casado-Mejía R, Botello-Hermosa A. Representatividad de las mujeres en las sociedades científicas en el ámbito de la salud en España en 2014. Gac Sanit. 2015;29(3):209-212.

20. Sindicato Médico Andaluz. Distribución de las peticiones de plazas MIR 2014. Granada: CESM; 2014. Disponible en: http:/www.cesm.org.es/images/009/ Informe-MIR-2014.pdf

21. Flores-Domínguez C. Feminización en Medicina: 1iderazgo y academia. Educ Med. 2012;15:191-5

22. Eisler R. El cáliz y la espada: la mujer como fuerza en la historia. México DF: Ed Pax México; 2005. 